translated into practice to provide safe and appropriate doses in children over the 91 st percentile for BMI (Body Mass Index)

Methods We chose 6 drugs (aciclovir, aminophylline, gentamicin, oromorph, paracetamol and phenytoin) that have particularly narrow therapeutic windows and a high risk of toxicity with suggested dosage adjustments based on ideal (IBW) or adjusted body weight (ABW) than actual body weight.

First a prescribing exercise was carried out using case scenarios in a group of paediatricians during a departmental teaching session in a tertiary children's hospital.

Next, over 4 weeks, all patients on the wards were screened for being overweight or obese. Children between the ages of 2 and 16 years with BMI over 91 st centile and on one or more of the listed drugs were included.

Results In the prescribing task we had 7 paediatricians from the level of Foundation Year 1 to Consultant. Out of the 42 individual question responses none were answered using the correct dose adjustment.

In the ward-based project, none of the 13 patients identified were prescribed the correct adjusted doses of their medications using IBW or ABW.

The maximum daily drug overdose was $149 \%$ for a prescription of paracetamol in an 11-year-old girl. The minimum daily drug overdose was $111 \%$. The average daily drug overdose from what was being prescribed for obese children on the ward in this four-week window was $128 \%$.

Conclusion We identified a lack of knowledge amongst paediatricians on prescribing in children with a $\mathrm{BMI} \geq 91$ st percentile although the sample size was small. There must be a call for more awareness and further studies to evaluate the mechanisms of altered pharmacokinetics in overweight and obese children to guide future drug dose adjustments and formulate universal guidelines.

\section{G92(P) 4-YEAR EXPERIENCE OF USING HIGH FLOW NASAL CANNULA (HFNC) OXYGEN THERAPY ON PAEDIATRIC WARDS}

F Roked, S Samar, D Jyothish. General Paediatrics, Birmingham Women's and Children's Hospital NHS Trust, Birmingham, UK

\subsection{6/archdischild-2018-rcpch.90}

We describe our experience of using High Flow Nasal Cannula (HFNC) Oxygen on the paediatric wards during a 4 year period from January 2014 to October 2017. A standard operating procedure (SOP) provided instructions and guidance for the use of HFNC. Retrospective analysis of demographic data, clinical conditions, indications, HFNC settings, adherence to SOP, adverse events and safety profile was undertaken over the 4 year period. HFNC was used in 124 babies and children, for a variety of conditions requiring respiratory assistance. Bronchiolitis was the commonest indication for the use of HFNC, followed by pneumonia, viral induced wheeze, asthma, and for alleviation of respiratory distress at the end of life in palliative care situations. Relatively high flow rates compared to other centres of upto 4 Litres $/ \mathrm{kg}$ was used, with no adverse effects such as air leaks. Conversion to NIV modalities such as CPAP and BiPap and intubation rates was comparable to other centres. Our findings agreed with previous studies that HFNC failure rates could be predicted by the lack of repsonse of respiratory distress and oxygen requirement, 60-90 min after commenicng HFNC. Incremental expansion of the use of HFNC was seen over the study period as well as extension of its use to a variety of clinical conditions. This was achieved without additional medical resources, but by nursing empowerment through training for the safe initiation, weaning and cessation of HFNC, as well as timely escalation.

Our experience of the use of HFNC is unique in using high flow rates in large numbers of children with a wide variety of respiratory conditions, in a non-paediatric intensive care setting.

\section{G93(P) SEPSIS: A CAUSE OF SUDDEN UNEXPECTED DEATH AND A WORKING DEFINITION}

${ }^{1} \mathrm{NT}$ Dlamini, ${ }^{1} \mathrm{~S}$ Band, ${ }^{1} \mathrm{~A}$ Tabor, ${ }^{1,2} \mathrm{M}$ Samuels. ${ }^{1}$ Royal Stoke University Hospital, University Hospitals of North Midlands, Stoke on Trent, UK; ${ }^{2}$ Walrus Respiratory Unit, Great Ormond Street Hospital, London, UK

\subsection{6/archdischild-2018-rcpch.91}

Background Some cases of sudden unexpected death in infants and children (SUDIC) occur from fulminant sepsis. There is no agreed definition for accepting this mechanism of death within SUDICs.

Aims To characterise the history, Emergency Department (ED) and post-mortem (PM) findings in children who died unexpectedly and in whom the likely diagnosis was sepsis and to make a working definition for Sudden Unexpected Death (SUDIC) due to Sepsis.

Methods We reviewed all SUDIC cases between 2008 and 2016 listed on the database for the local Child Death Overview Panel, and identified those thought to be due to natural causes. We then collected clinical details alongside the ED and PM findings to characterise which deaths were likely to be due to severe and overwhelming infection. Our early management of SUDIC includes the collection of samples as soon as possible after death for investigation of infection.

Results Over 8 years, there were 160 cases designated as SUDIC due to natural causes, of which 17 (11\%) were from infection-related causes. We examined complete records for 12 children ( 7 girls), aged 4 months to 12 years (median 26 months). Three (25\%) had preceding signs suggestive of infection, but insufficient to diagnose sepsis. Five of the other 9 children died from beta haemolytic group A streptococcus sepsis, one from beta haemolytic group B streptococcus sepsis, one from staphylococcus aureus sepsis, and one from Haemophilus influenza sepsis. In the remaining child, the cause of death was unascertained but 'likely sepsis secondary to streptococcal infection'.

Eight children were unwell for 48 hours, with a median duration of symptoms of 24 hours. Six had medical review before death. Symptoms included: fever, cough, coryza, unsettled, lethargy, vomiting and diarrhoea and all but one were managed as viral illnesses.

Conclusion Lethal sepsis can progress rapidly, but be difficult to distinguish from viral infection at onset. A working definition for SUDIC-sepsis includes: sudden death with i) prodromal symptoms; ii) a likely pathogen in a normally sterile site; and, iii) evidence of inflammatory reaction (such as raised CRP, WCC and histological changes). 